\title{
New optics for resolution improving of Ring Imaging Cherenkov detectors
}

\author{
M. Šulc ${ }^{1,2, a}$, D. Kramer $^{3}$, J. Polak ${ }^{2}$, L. Steiger ${ }^{2,4}$, M. Finger ${ }^{5}$, M. Slunecka ${ }^{5}$ \\ ${ }^{1}$ Technical University of Liberec, Department of Physics, Studentska 2, 46117 Liberec, Czech Republic \\ ${ }^{2}$ Research Centre for Special Optics and Optoelectronic Systems (TOPTEC), Institute of Plasma Physics, AS CR, \\ Sobotecka 1660, 51101 Turnov, Czech Republic \\ ${ }^{3}$ Institute of Physics, AS CR, Na Slovance 1999/2, 18221 Praha 8, Czech Republic \\ ${ }^{4}$ Institute of Mechatronics and Technical Engineering, Technical University of Liberec, Studentska 2, 46117 Liberec, \\ Czech Republic \\ ${ }^{5}$ Charles University Prague, Faculty of Mathematics and Physics, Ke Karlovu 3, 121 16, Prague 2, Czech Republic and \\ JINR, Dubna, Russia
}

\begin{abstract}
The Ring Imaging Cherenkov detector (RICH) of the COMPASS experiment at CERN is key tool for particle identification. Two reflecting spherical mirror surfaces, covering a total area of about $21 \mathrm{~m}^{2}$, hosted in the radiator vessel, provide Cherenkov radiation focusing to photon detectors. These ones are based on the use of multi-anode photo-multiplier tubes. They are coupled to individual lens telescopes, made from special fused silica aspherical lenses. Design, construction, and Hartmann test of lenses qualities and alignment were described. The RICH detector uses $\mathrm{C}_{4} \mathrm{~F}_{10}$ as radiator gas. The refractive index of the radiator gas is substantial parameter. It varies with temperature, atmospheric pressure and gas purity. Its accurate knowledge is essential for the particle identification performance. A modified Jamin's interferometer was proposed, constructed and tested to allow on-line refractive index measurement with accuracy better than $10^{-6}$. The new types of fused silica Cherenkov radiators was designed to the tests of electron multiplier detector too.
\end{abstract}

\section{Introduction}

The high-energy COMPASS experiment [1] is a fixed-target experiment at CERN SPS that uses a muon beam to study nucleon spin structures, and hadron beams for hadron spectroscopy research. An essential element of the COMPASS spectrometer [2] is the ability to perform hadron identification in a wide range of particle momenta at high rates and over a large angular acceptance. This capability is provided by gaseous Ring Imaging Cherenkov detector - COMPASS RICH-1.

This detector is the most complex one at COMPASS experiment. It uses a lot of optical elements and methods for particle identification. It is using $80 \mathrm{~m}^{3}$ of $\mathrm{C}_{4} \mathrm{~F}_{10}$ radiator, a $21 \mathrm{~m}^{2}$ large wall of spherical mirrors and a 5.5 $\mathrm{m}^{2}$ large photon-detector area. Multi-wire proportional chambers (MWPC) with CsI photocathodes are used for the detection of Cherenkov photons in the peripheral region ( $75 \%$ of the total area). The central part of detector, covering $25 \%$ of total area, was equipped by 576 multianode $(4 \times 4)$ photomultiplier tubes (MAPMT) during the upgrade [3]. The MAPMTs are coupled to

\footnotetext{
a e-mail: sulc@ipp.cas.cz
}

individual telescopes of fused silica lenses to enlarge the effective detection area.

One of the most important characteristics of Ring Imaging Cherenkov detectors is the refractive index of the radiator. Its accurate knowledge is essential for particle velocity measurement and particle identification. Both the absolute values at specific wavelengths and the dispersion of the refractive index significantly influence the precision of a particle momentum measurement. A modified Jamin interferometer was proposed, constructed and tested to allow on-line refractive-index measurement.

The new types of detector, thick gaseous electron multipliers (TH-GEM), were tested with the aim to replace CsI photodiodes. It was necessary to develop and produce the set of fused silica Cherenkov radiators for the detector tests with particle beams.

\section{The lens telescope}

The spherical mirrors have focused light into Cherenkov ring in detector plane. The previous detectors MWPCs were replaced by PMTs ones in part of this plane. It is why two-lens telescope was designed to

This is an Open Access article distributed under the terms of the Creative Commons Attribution License 2.0, which permits unrestricted use, distribution, and reproduction in any medium, provided the original work is properly cited. 
concentrate the light to the MAPMT photocathode, preserving information about position of Cherenkov photon at detector plane [3]. Two constrains come from the existing COMPASS spectrometer set-up. The total telescope length cannot exceed $15 \mathrm{~cm}$, due to the reduced space available upstream of RICH-1 in the COMPASS spectrometer. The MAPMT has to be oriented at an angle with respect to the telescope entrance axis, a feature needed for compatibility with the existing MWPCs that remain as peripheral photon detectors. It results to condition, that the telescope has to be a non-axial system.

This required telescope system is not-imaging system. Different designs in Zemax ${ }^{\odot}$ optical software were made together with Monte Carlo simulations. The design was at first optimized for the best performance and then its imaging quality was decreased in steps to match the given mechanical and production cost restraints. The specific figure of merit was built to compare the results of the simulations, and taking into account all specific problems of $\mathrm{RICH}-1$ detector. Optimal solution was compromise between fraction of the generated photons, collected by the detector, and the fraction of the collected photons, concentrated on the correct pixel.

The telescope was made from front plano-convex lens with the rectangular aperture $48 \times 42 \mathrm{~mm}^{2}$, radius of curvature $R_{1}=54.937 \mathrm{~mm}$, and second one with the first aspherical optical surface, $R_{3}=20.696 \mathrm{~mm}$, and the aspherical $4^{\text {th }}$ order coefficient of the third surface $\alpha=-$ $6.13884 \cdot 10^{-5} \mathrm{~mm}^{-3}, R_{4}=-24.964 \mathrm{~mm} . T_{1}=20.813 \mathrm{~mm}$, $T_{2}=75.805 \mathrm{~mm}, T_{3}=19.420 \mathrm{~mm}$ and $T_{4}=26.835 \mathrm{~mm}$ are the distances between the surfaces, $D=34.68 \mathrm{~mm}$ is the diameter of the second lens; see figure 1.

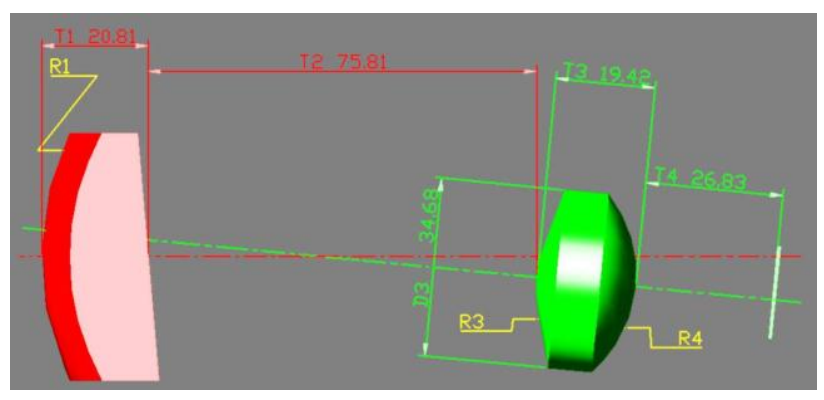

Fig. 1. The side view of final design of the telescope

A sensitivity study was performed resulting in production tolerances for the lenses and their mounting system. The designed system was produced in 600 pieces.

The lenses have been evaluated using the Hartmann test. The comparison between the spot position in the image and the expected position for the ideal optical object was used. The accepted tolerance for the optical object can be translated in an area around the ideal position. If the actual spot is contained in this area, the optical distortions are within tolerances, see figure 2.

Quality of prototype lenses, in parallel, was measured also with interferometric methods. To perform the quality control of the whole telescopes, each complete structure was at first assembled without introducing the
MAPMTs in the iron boxes. It was so possible to use the Hartmann setup, because the image can be collected downstream of the telescope. The method used the same apparatus for the quality control of the alignment of the lenses, glued to the field lens frame, namely the Hartmann setup is fixed, while the complete panel is moved by a milling machine. Finally the telescope imperfections were tested too. They are due to imperfections of the concentrator lens holders, made by soft iron. This material, required to form a magnetic screen around the MAPMT, is not ideal for precise machining. When small telescope imperfections are detected, it was possible to correct for them. The corrections are obtained by tilting the concentrator lens with respect to the MAPMT frame, to which the complete soft iron box is screwed.

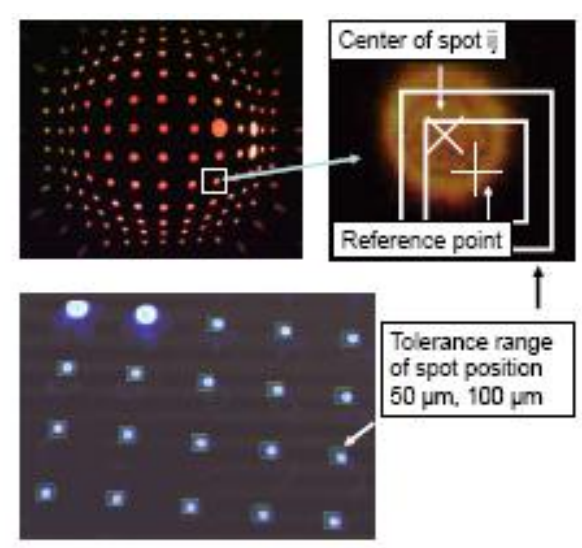

Fig. 2. Typical image collected with the Hartmann setup. The zoom of a spot is shown.

After applying of these corrections, $70 \%$ of the telescopes exhibit a small deviation of detected photon position on PMT plane (with respect to the ideal one) below $50 \mu \mathrm{m}, 20 \%$ between $50-100 \mu \mathrm{m}$ and $10 \%$ between $100-150 \mu \mathrm{m}$.

\section{Jamin interferometer}

Accurate knowledge of refractive index of $\mathrm{C}_{4} \mathrm{~F}_{10}$, with accuracy of better than $10^{-5}$, is essential for the particle identification. The modified folded Jamin interferometer [4], insensitive to the rotation and translation of its two optical elements was proposed, constructed and tested [5]. It is relatively simple and vibration-insensitive. An incoming $\mathrm{He}-\mathrm{Ne}$ laser beam $(633 \mathrm{~nm})$ is split by a polarization-sensitive partial beamsplitter coating on the second surface of a plane-parallel plate. One of the resulting beams is reflected by a highreflectance coating on the first surface. Two parallel beams of equal intensity with orthogonal polarizations are generated by this beam-splitter coating. The first beam passes through a closed quartz pipe (evacuated or gas-filled); the second beam passes outside this pipe through the measured gas, see figure 3 . Both beams are reflected by a retro-reflecting prism, and they pass 
through the same quartz disk on the end of the pipe, so the phase difference between the two beams is only induced by the refractive-index difference.

The beams are joined together and reflected by an additional mirror into the special detector unit, which divides the signal into two detectors placed behind two analyzers (rotated by $+45^{\circ}$ and $-45^{\circ}$ with respect to the first beam polarization). The two signals with an exact $\pi / 2$ relative phase shift are measured. This technique enables the measurement of not only the number of passing interferometric fringes but also the direction of their movement and the phase differences smaller than $2 \pi$ (quadrature encode measurement).

The interferometer is placed inside a stainless steel chamber to protect the He-Ne laser from the external magnetic field. The interferometer is directly connected to the COMPASS RICH-1 vessel. The pressure and temperature control detectors inside the chamber verify that the gas conditions are the same as those inside the $\mathrm{RICH}$ vessel.

The measurement starts with the interferometer pipe filled with the $\mathrm{C}_{4} \mathrm{~F}_{10}$ gas; there is no difference between the beam phases. The pipe is connected to a vacuum pump by an electromagnetic valve and is slowly evacuated. The fringes (about 1400) move over the interferometer photodetectors. This setup allows the measurement of the absolute value of the gas refractive index (with respect to the vacuum). Changes of phase difference, caused by refractive index instabilities, are observed afterwards. The interferometer sensitivity is excellent. The accuracy of one fringe movement measurement (equivalent to a $2 \pi$ phase difference) corresponds to a change of $10^{-6}$ in the refractive index. This measurement can be further improved by a factor of 100 using the appropriate software. A completely automatic driving system is foreseen. At regular time intervals (and in case of laser or vacuum instability) the initial measurement will be repeated by filling the interferometer pipe with gas and evacuating this pipe using the electromagnetic valve set, driven by a PLC.

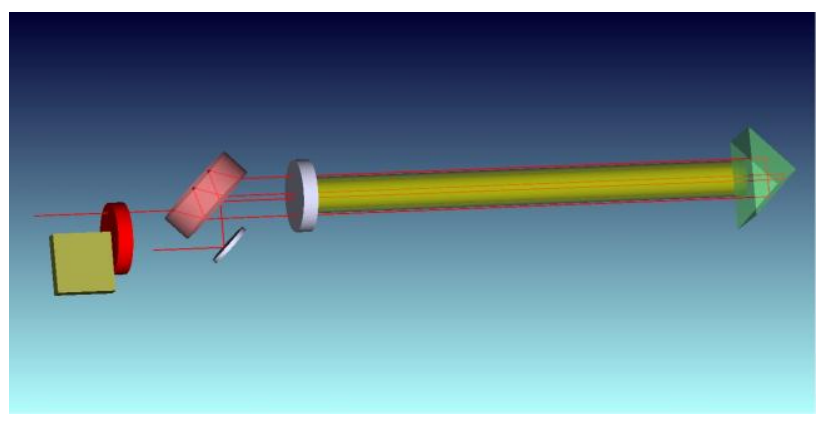

Fig. 3. Folded Jamin interferometer for measurement of gas refractive index.

\section{Cherenkov radiators}

The Cherenkov radiators were developed for testing of new sensitive photon detectors - thick gaseous electron multipliers (TH-GEM). The design of radiators was made by Zamax $^{(}$software, where problems with simulation of source and propagation of Cherenkov radiation was solved. Different types for various purposes were designed [6]. Hemispherical radiator can focus radiation to narrow ring. Long radiator can produce very large amount of photons, proportional to the radiator length, due a specific property of Cherenkov radiation. Particle beam, going in direction of a cylinder axis, produces Cherenkov photons, falling to the cylinder surface in critical angle. So photons are completely reflected. All radiators were produced and used in particle beam tests of TH-GEMs.

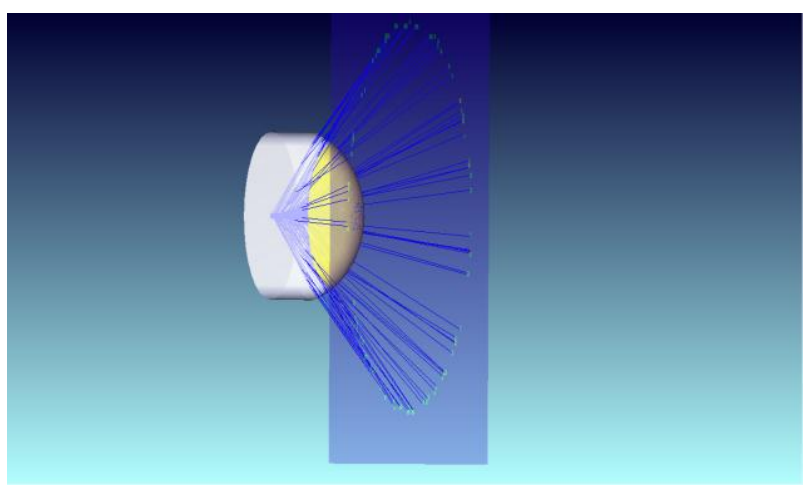

Fig. 4. Hemispherical fused silica radiator, producing ring of Cherenkov photons.

New type of radiator, using aspherical surface, was designed. It can be produced by new machining technologies. It consists of significantly smaller amount of fused silica, and can focus Cherenkov radiation to sharp ring of required properties.

\section{Conclusion}

New optical instrument and methods, used for Ring Imaging Cherenkov detector at Compass experiment at CERN, helped to improve sensitivity of apparatus for particle identification.

\section{Acknowledgments}

This work was partially supported by the European Regional Development Fund and the MEYS of the Czech Republic in the Project No. CZ.1.05/2.1.00/03.0079: Research Center for Special Optics and Optoelectronic Systems (TOPTEC) and MEYS project LA08015.

\section{References}

1. P. Abbon et al., Nucl. Inst. Meth. A 577, 455-518. (2007)

2. E. Albrecht, et al., Nucl. Instr. and Meth. A 553, 215-219 (2005)

3. P. Abbon et al., Nucl. Inst. Meth. A 616, 21-37 (2010)

4. A.J. Sedlacek, REVIEW OF SCIENTIFIC INSTRUMENTS 77, 064903 (2006) 
5. S. Dalla Torre et al., Nucl. Instr. and Meth. A 639, 271-273 (2011)

6. M.Sulc, Jemná mechanika a optika(Fine mechanics and optics) 56 [9], 247/250, (2011) 